\title{
Scotogenic Neutrino Masses and Dark Matter Stability from Residual Gauge Symmetry
}

\author{
Julio Leite ${ }^{*},{ }^{1,2}$ Oleg Popov, ${ }^{3,4}$ Rahul Srivastava, ${ }^{1,5}$ and José W. F. Valle ${ }^{1}$ \\ ${ }^{1}$ AHEP Group, Institut de Física Corpuscular - C.S.I.C./Universitat de València, Parc Científic de Paterna. \\ C/ Catedrático José Beltrán, 2 E-46980 Paterna (Valencia) - SPAIN \\ ${ }^{2}$ Centro de Ciências Naturais e Humanas, Universidade Federal do ABC, 09210-580, Santo André-SP, Brasil \\ ${ }^{3}$ Institute of Convergence Fundamental Studies, Seoul National University of Science and Technology, \\ Seoul 139-743, Republic of Korea \\ ${ }^{4}$ Department of Physics, Korea Advanced Institute of Science and Technology, \\ 291 Daehak-ro, Yuseong-gu, Daejeon 34141, Republic of Korea \\ ${ }^{5}$ India Institute of Science Education and Research - Bhopal, Bhopal Bypass Road, Bhauri, 462066, Bhopal, India
}

\begin{abstract}
In the context of the $\mathrm{SU}(3)_{\mathrm{C}} \otimes \mathrm{SU}(3)_{\mathrm{L}} \otimes \mathrm{U}(1)_{\mathrm{X}} \otimes \mathrm{U}(1)_{\mathrm{N}}$ (3-3-1-1) extension of the standard model, we show how the spontaneous breaking of the gauge symmetry gives rise to a residual symmetry which accounts for dark matter stability and small neutrino masses in a scotogenic fashion. As a special feature, the gauge structure implies that one of the light neutrinos is massless and, as a result, there is a lower bound for the $0 v \beta \beta$ decay rate.
\end{abstract}

Keywords: Beyond the Standard Model, Neutrino masses, Dark matter

DOI: 10.31526/ACP.NDM-2020.22

\section{INTRODUCTION}

The lack of a viable dark matter (DM) candidate within the Standard Model is one of the most pressing issues requiring new physics. In addition to new particles, the existence of DM requires new symmetries to stabilise the corresponding candidate on cosmological scales, e.g. R-parity symmetry in supersymmetric schemes [1]. Another important open question in need of new physics is that of neutrino masses, which are necessary to account for neutrino oscillation data [2].

In order to deal simultaneously with the DM and neutrino mass issues, "low-scale" realisations where dark matter appears as a radiative mediator of neutrino mass generation, known as scotogenic models, are appealing alternatives. In its original version [3], the symmetry stabilising dark matter is also responsible for the radiative origin of neutrino masses in a very elegant way. Yet, in this case as well as in other proposals [4,5], the stabilisation symmetry is introduced in an ad hoc manner.

Extending the Standard Model gauge symmetry can provide a natural setting for a theory of dark matter where stabilisation is automatic $[6,7,8]$. Such electroweak extensions involve the $\mathrm{SU}(3)_{\mathrm{L}}$ gauge symmetry, which remarkably explains the observed number of fermion families from the anomaly cancellation requirement $[9,10,11]$. In the extended models discussed in $[6,7,8]$, DM stability follows naturally from the spontaneous breaking of the extended gauge symmetry into the residual matter-parity symmetry, $M_{P}$, a non-supersymmetric version of R-parity.

We show here how scotogenic neutrino masses and automatic DM stabilisation are intrinsically linked in a 3-3-1-1 model [12]. The present construction has the special feature of predicting that one of the light neutrinos is massless, which, in turn, implies that there is a lower bound for neutrinoless double beta decay rate. This feature arises in a novel way when compared to other schemes in the literature, such as the "incomplete" seesaw mechanism [13] or similar radiative mechanisms [14].

\section{THE MODEL}

In our model [12], the electric charge and $B-L$ generators are given by $Q=T_{3}-(1 / \sqrt{3}) T_{8}+X$ and $B-L=-(2 / \sqrt{3}) T_{8}+N$, respectively, where $T_{3}$ and $T_{8}$ are the diagonal $S U(3)_{L}$ generators, while $X$ and $N$ are the $U(1)_{X}$ and $U(1)_{N}$ generators, respectively. Notice that, due to the extra $U(1)_{N}$ symmetry, the $B-L$ symmetry is fully gauged. The lepton and scalar fields and their respective transformations are summarised in Table 1. In the lepton sector, the left-handed fields appear as $S U(3)_{L}$ triplets, while the righthanded are $S U(3)_{L}$ singlets. The scalar sector contains five triplets and one singlet.

Symmetry breaking takes place when scalar fields acquire vacuum expectation values (vevs), according to the pattern below,

$$
\langle\sigma\rangle=\frac{v_{\sigma}}{\sqrt{2}}, \quad\langle\chi\rangle=\frac{1}{\sqrt{2}}(0,0, w)^{T},\langle\eta\rangle=\frac{1}{\sqrt{2}}\left(v_{1}, 0,0\right)^{T}, \quad\langle\rho\rangle=\frac{1}{\sqrt{2}}\left(0, v_{2}, 0\right)^{T}, \quad\langle\zeta\rangle=\frac{1}{\sqrt{2}}\left(0, v_{2}^{\prime}, 0\right)^{T} .
$$




\begin{tabular}{|c|c|c|c|c|c|c|}
\hline Field & $\mathrm{SU}(3)_{L}$ & $\mathrm{U}(1)_{X}$ & $\mathrm{U}(1)_{N}$ & $Q$ & $B-L$ & $M_{P}=(-1)^{3(B-L)+2 s}$ \\
\hline \hline$l_{a L}$ & $\mathbf{3}$ & $-1 / 3$ & $-2 / 3$ & $(0,-1,0)^{T}$ & $(-1,-1,0)^{T}$ & $(++-)^{T}$ \\
$e_{a R}$ & $\mathbf{1}$ & -1 & -1 & -1 & -1 & + \\
\hline$v_{i R}$ & $\mathbf{1}$ & 0 & -4 & 0 & -4 & + \\
$v_{3 R}$ & $\mathbf{1}$ & 0 & 5 & 0 & 5 & - \\
$N_{a R}$ & $\mathbf{1}$ & 0 & 0 & 0 & 0 & $(++-)^{T}$ \\
\hline \hline$\eta$ & 3 & $-1 / 3$ & $1 / 3$ & $(0,-1,0)^{T}$ & $(0,0,1)^{T}$ & $(++-)^{T}$ \\
$\rho$ & 3 & $2 / 3$ & $1 / 3$ & $(1,0,1)^{T}$ & $(0,0,1)^{T}$ & $(--+)^{T}$ \\
$\chi$ & 3 & $-1 / 3$ & $-2 / 3$ & $(0,-1,0)^{T}$ & $(-1,-1,0)^{T}$ & + \\
$\sigma$ & $\mathbf{1}$ & 0 & 2 & 0 & 2 & $(+,+,-)^{T}$ \\
\hline$\zeta$ & 3 & $2 / 3$ & $7 / 3$ & $(1,0,1)^{T}$ & $(2,2,3)^{T}$ & $(-,-,+)^{T}$ \\
\hline
\end{tabular}

TABLE 1: Lepton and scalar content with $a=1,2,3$ and $i=1,2$.

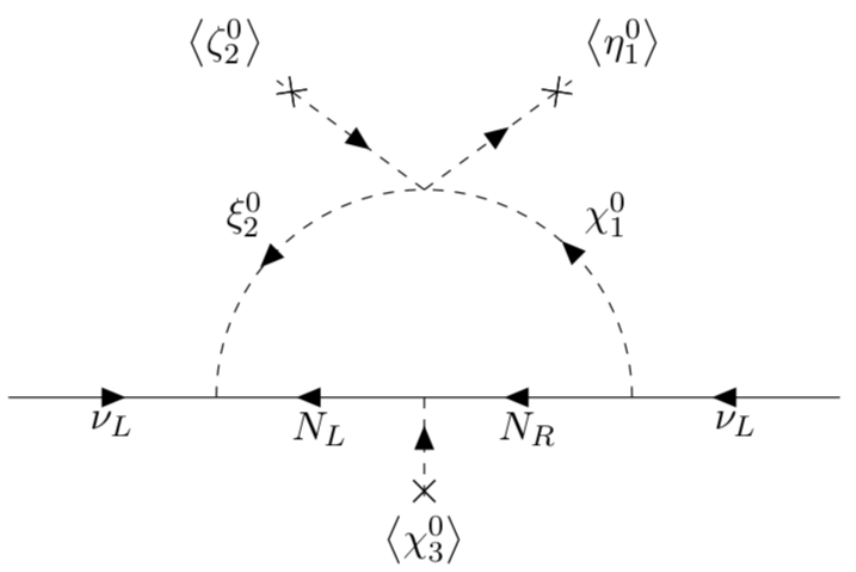

FIGURE 1: 1-loop "scotogenic" neutrino mass.

The spontaneous symmetry breaking process can be divided into two main steps

$$
S U(3)_{c} \otimes S U(3)_{L} \otimes U(1)_{X} \otimes U(1)_{N} \stackrel{v_{\sigma}, w}{\longrightarrow} S U(3)_{\mathcal{C}} \otimes S U(2)_{L} \otimes U(1)_{Y} \otimes M_{P} \stackrel{v_{1}, v_{2}, v_{2}^{\prime}}{\longrightarrow} S U(3)_{c} \otimes U(1)_{Q} \otimes M_{P}
$$

for $v_{\sigma}, w \gg v_{E W}$ and $v_{E W}=\left(v_{1}^{2}+v_{2}^{2}+v_{2}^{\prime 2}\right)^{1 / 2}=246 \mathrm{GeV}$. After the breaking a residual matter-parity symmetry, $M_{P}$, is present

$$
M_{P}=(-1)^{3(B-L)+2 s},
$$

where $s$ is the particle's spin. As only the $M_{P}$-even scalar fields get vevs, $M_{P}$ remains as an absolutely conserved residual symmetry, in such a way that the lightest amongst the $M_{P}$-odd particles is stable, see Table 1.

Taking into account the scalar fields in Table 1, we can write down the most general renormalisable potential as

$$
\begin{aligned}
V= & \sum_{s}\left[\mu_{s}^{2}\left(s^{\dagger} s\right)+\frac{\lambda_{s}}{2}\left(s^{\dagger} s\right)^{2}\right]+\sum_{s_{1}, s_{2}}^{s_{1}>s_{2}}\left[\lambda_{s_{1} s_{2}}\left(s_{1}^{\dagger} s_{1}\right)\left(s_{2}^{\dagger} s_{2}\right)\right]+\sum_{t_{1}, t_{2}}^{t_{1}>t_{2}}\left[\lambda_{t_{1} t_{2}}^{\prime}\left(t_{1}^{\dagger} t_{2}\right)\left(t_{2}^{\dagger} t_{1}\right)\right]+\frac{\mu_{1}}{\sqrt{2}} \eta \rho \chi+\frac{\mu_{2}}{\sqrt{2}}\left(\zeta^{\dagger} \rho\right) \sigma \\
& +\lambda_{1}\left(\chi^{\dagger} \eta\right)\left(\zeta^{\dagger} \xi\right)+\lambda_{2}\left(\chi^{\dagger} \xi\right)\left(\zeta^{\dagger} \eta\right)+\lambda_{3}\left(\chi^{\dagger} \eta\right)\left(\xi^{\dagger} \rho\right)+\lambda_{4}\left(\chi^{\dagger} \rho\right)\left(\xi^{\dagger} \eta\right)+\lambda_{5}(\eta \zeta \chi) \sigma^{*}+\text { h.c. },
\end{aligned}
$$

where $s=\eta, \rho, \chi, \sigma, \zeta, \xi$ (all scalars), and $t=\eta, \rho, \chi, \zeta, \xi$ (scalar triplets only).

\subsection{Neutrino masses}

When it comes to the Yukawa interactions, we can write the Lagrangian below

$$
-\mathcal{L}_{\text {lep }}=y_{a b}^{e} \overline{l_{a L}} \rho e_{b R}+y_{a b}^{N} \overline{l_{a L}} \chi N_{b R}+h_{a b} \overline{l_{a L}}\left(l_{b L}\right)^{c} \xi^{*}+\frac{\left(m_{N}\right)_{a b}}{2} \overline{\left(N_{a R}\right)^{c}} N_{b R}+\text { h.c. },
$$

where $y^{e}, y^{N}, h$ and $m_{N}$ are complex $3 \times 3$ matrices, with $m_{N}$ being symmetric due to the Pauli principle. In contrast, $h$, the Yukawa coupling which governs the anti-symmetric contraction of three triplets, is necessarily anti-symmetric in family space. 


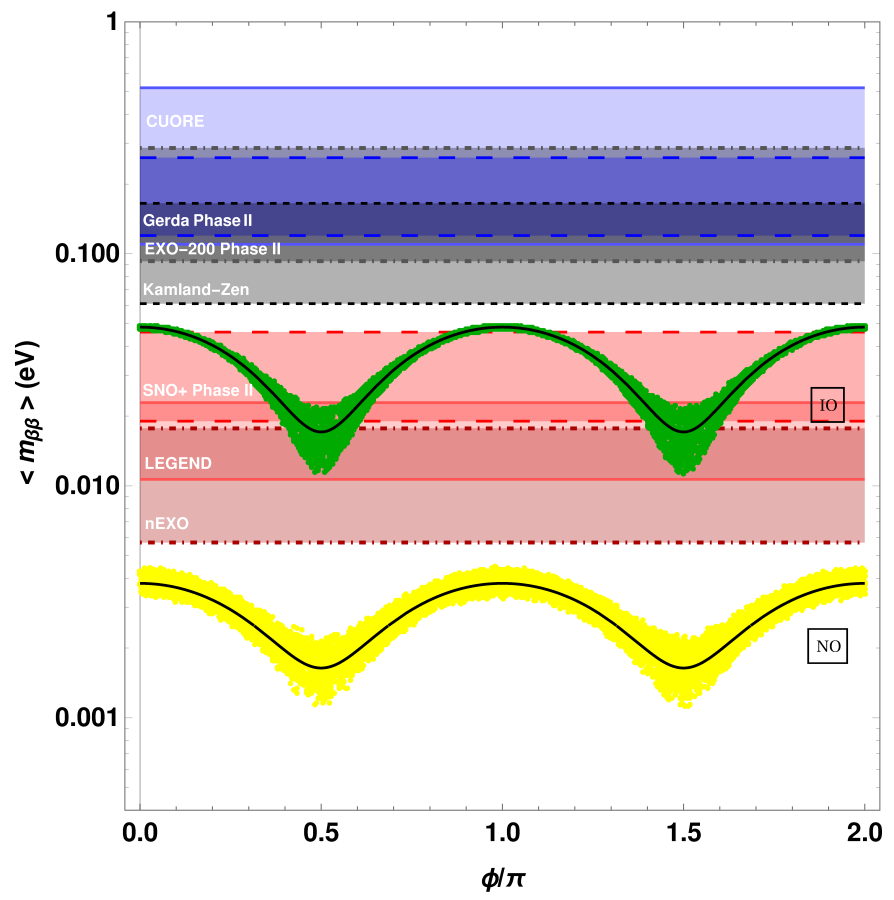

FIGURE 2: Effective Majorana mass vs relative Majorana phase for the case of inverted (green) and normal (yellow) mass ordering. Experimental limits and future sensitivities are displayed as horizontal bands.

Charged lepton masses come from the first term in Eq. (5) when $\rho$ acquires a vev: $M^{e}=y^{e} v_{2} / \sqrt{2}$. The neutral leptons $N_{i L}$ and $N_{i R}$ mix at tree-level when $\chi$ acquires a vev, and the mixing angle is defined by $\tan \left(2 \theta_{N}\right)=2 y^{N_{w}} / m_{N}$.

Let us now turn our attention to the active neutrinos $v_{L}$. As neither the first component of $\chi$ nor the second of $\xi$, both $M_{P}$-odd, acquires a vev, neutrinos are massless at tree-level. Nevertheless, neutrino masses are radiatively generated via the loop diagram in Fig.1.

In order to calculate this diagram, we go from the flavour basis where the internal scalar fields, $\chi_{1}^{0}$ and $\xi_{2}^{0}$, mix with each other as well as with $\eta_{3}^{0}$, to the physical basis. The physical fields are three $\mathrm{CP}$-even and three $\mathrm{CP}$-odd neutral scalars, $(S, A)_{1,2,3}$, which mix according to two mixing angles, $\left(\theta_{S, A}\right)_{1,2}$, as shown in Ref. [12]. Thus, the one-loop neutrino masses can be written as

$$
\begin{aligned}
m_{v}^{a b} & =\frac{1}{8 \pi^{2}} h^{* a c} s_{N} c_{N} c_{1}\left\{m_{N_{1}}\left[s_{S_{2}} c_{S_{2}}\left(Z\left(\frac{m_{S_{1}}^{2}}{m_{N_{1}}^{2}}\right)-Z\left(\frac{m_{S_{2}}^{2}}{m_{N_{1}}^{2}}\right)\right)-s_{A_{2}} c_{A_{2}}\left(Z\left(\frac{m_{A_{1}}^{2}}{m_{N_{1}}^{2}}\right)-Z\left(\frac{m_{A_{2}}^{2}}{m_{N_{1}}^{2}}\right)\right)\right]\right. \\
& \left.-m_{N_{2}}\left[s_{S_{2}} c_{S_{2}}\left(Z\left(\frac{m_{S_{1}}^{2}}{m_{N_{2}}^{2}}\right)-Z\left(\frac{m_{S_{2}}^{2}}{m_{N_{2}}^{2}}\right)\right)-s_{A_{2}} c_{A_{2}}\left(Z\left(\frac{m_{A_{1}}^{2}}{m_{N_{2}}^{2}}\right)-Z\left(\frac{m_{A_{2}}^{2}}{m_{N_{2}}^{2}}\right)\right)\right]\right\} y_{c d}^{N * d b}+\{a \leftrightarrow b\},
\end{aligned}
$$

with $s_{x} \equiv \sin \theta_{x}, c_{x} \equiv \cos \theta_{x}$ and $Z(x)=\frac{x}{1-x} \ln x$. The antisymmetryic nature of the Yukawa matrix $h$ implies that the neutrino mass matrix is of rank two, leading to one massless neutrino. This unique feature provides a novel origin for the masslessness of one neutrino to be contrasted with the usual models relying on missing partner mechanisms.

All fields running inside the neutrino mass loop are odd under the exactly conserved matter-parity symmetry. Therefore, the lightest among such particles, either a scalar or a fermion, is stable and can play the role of a WIMP dark matter.

\subsection{Neutrinoless double beta decay}

The model predicts Majorana neutrinos, which allows for the possibility of neutrinoless double beta $(0 v \beta \beta)$ decay to take place [15]. The dominant contribution to $0 v \beta \beta$ decay is the standard one and depends on the effective Majorana mass, defined as

$$
\left\langle m_{\beta \beta}\right\rangle=\left|\cos ^{2} \theta_{12} \cos ^{2} \theta_{13} m_{1}+\sin ^{2} \theta_{12} \cos ^{2} \theta_{13} m_{2} e^{2 i \phi_{12}}+\sin ^{2} \theta_{13} m_{3} e^{2 i \phi_{13}}\right|,
$$

where mixing angles and Majorana phases are neatly expressed in the symmetric parametrisation of the lepton mixing matrix [13], and the neutrino masses $m_{a}$ obtained from Eq. 6 .

It is well-known that, in a generic model, this amplitude can vanish for normal-ordered neutrinos, currently preferred by oscillations [2]. However, in our model, since one neutrino is massless, $\left\langle m_{\beta \beta}\right\rangle$ never vanishes, as shown in Fig. 2. 


\section{CONCLUSIONS}

We have proposed an $\mathrm{SU}(3)_{\mathrm{c}} \otimes \mathrm{SU}(3)_{\mathrm{L}} \otimes \mathrm{U}(1)_{\mathrm{X}} \otimes \mathrm{U}(1)_{\mathrm{N}}$ model where dark matter stability follows from the spontaneous breaking of the gauge symmetry. Neutrino masses are generated at loop level and mediated by DM in a scotogenic fashion. Our construction features a triplet scalar with anti-symmetric Yukawa couplings to neutrinos. This leads to the prediction of a massless neutrino and a lower bound for the $0 v \beta \beta$ decay rate. Contrary to models where a massless neutrino arises from an ad hoc incomplete multiplet choice, here it is an unavoidable feature of the theory.

\section{ACKNOWLEDGEMENTS}

Work supported by the Spanish grants SEV-2014-0398 and FPA2017-85216-P (AEI/FEDER, UE), PROMETEO/2018/165 (Generalitat Valenciana) and the Spanish Red Consolider MultiDark FPA2017-90566-REDC. J. L. acknowledges financial support under grant 2019/04195-7, São Paulo Research Foundation (FAPESP), while OP is supported by the National Research Foundation of Korea, under Grants No. 2017K1A3A7A09016430 and No. 2017R1A2B4006338.

\section{References}

[1] Gerard Jungman, Marc Kamionkowski, and Kim Griest. Supersymmetric dark matter. Phys. Rept., 267:195-373, 1996.

[2] P. F. de Salas, D. V. Forero, C. A. Ternes, M. Tortola, and J. W. F. Valle. Status of neutrino oscillations 2018: $3 \sigma$ hint for normal mass ordering and improved CP sensitivity. Phys. Lett., B782:633-640, 2018.

[3] Ernest Ma. Verifiable radiative seesaw mechanism of neutrino mass and dark matter. Phys. Rev., D73:077301, 2006.

[4] M. Hirsch, R. A. Lineros, S. Morisi, J. Palacio, N. Rojas, and J. W. F. Valle. WIMP dark matter as radiative neutrino mass messenger. JHEP, 10:149, 2013.

[5] Alexander Merle, Moritz Platscher, Nicolás Rojas, José W. F. Valle, and Avelino Vicente. Consistency of WIMP Dark Matter as radiative neutrino mass messenger. JHEP, 07:013, 2016.

[6] Alexandre Alves, Giorgio Arcadi, P. V. Dong, Laura Duarte, Farinaldo S. Queiroz, and José W. F. Valle. Matter-parity as a residual gauge symmetry: Probing a theory of cosmological dark matter. Phys. Lett., B772:825-831, 2017.

[7] P. V. Dong, D. T. Huong, Farinaldo S. Queiroz, José W. F. Valle, and C. A. Vaquera-Araujo. The Dark Side of Flipped Trinification. JHEP, 04:143, 2018.

[8] Sin Kyu Kang, Oleg Popov, Rahul Srivastava, José W. F. Valle, and Carlos A. Vaquera-Araujo. Scotogenic dark matter stability from gauged matter parity. Phys. Lett., B798:135013, 2019.

[9] M. Singer, J. W. F. Valle, and J. Schechter. Canonical Neutral Current Predictions From the Weak Electromagnetic Gauge Group SU(3) X u(1). Phys. Rev., D22:738, 1980.

[10] F. Pisano and V. Pleitez. An SU(3) x U(1) model for electroweak interactions. Phys. Rev., D46:410-417, 1992.

[11] P. H. Frampton. Chiral dilepton model and the flavor question. Phys. Rev. Lett., 69:2889-2891, 1992.

[12] Julio Leite, Oleg Popov, Rahul Srivastava, and José W. F. Valle. A theory for scotogenic dark matter stabilised by residual gauge symmetry. Phys. Lett., B802:135254, 2020.

[13] J. Schechter and J. W. F. Valle. Neutrino Masses in SU(2) x U(1) Theories. Phys. Rev., D22:2227, 1980.

[14] M. Reig, D. Restrepo, J. W. F. Valle, and O. Zapata. Bound-state dark matter with Majorana neutrinos. Phys. Lett., B790:303-307, 2019.

[15] J. Schechter and J. W. F. Valle. Neutrinoless Double beta Decay in SU(2) x U(1) Theories. Phys. Rev., D25:2951, 1982. 